\title{
Profesionalización del profesorado indígena. Retos en la Universidad Pedagógica Nacional, Unidad 071, de Chiapas ${ }^{1}$
}

\author{
Profissionalização de professores indígenas. Desafios da \\ Universidade Pedagógica Nacional, Unidade 071, de Chiapas \\ Professionalization of indigenous teachers. Challenges at the \\ National Pedagogical University, Unit 071, Chiapas
}

\section{Xürümtugen ta pu mapuche kimeltuchefe. Universidad Pedagógica Nacional ñi mülezuamüngen, 071 Xokitun, Chiapas Mapu.}

Recepción: 25/03/2019

Evaluación: 15/05/2019

Aceptación: 23/05/2019

Artículo de Investigación

DOI: https://doi.org/10.19053/01227238.10116

\author{
José Bastiani Gómez ${ }^{2}$ \\ Universidad Intercultural de Chiapas (UNICH), México
}

María Minerva López García ${ }^{3}$

Universidad Autónoma de Chiapas (UNACH), México

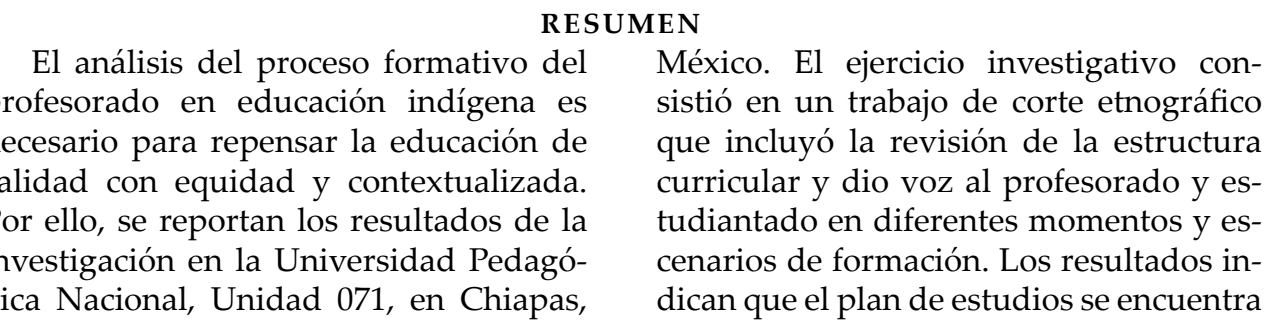

$1 \quad$ El artículo es resultado parcial del proyecto de investigación "El proceso de vinculación comunitaria como eje educativo integrador y sus aportes a la interculturalidad y la sustentabilidad en la Universidad Intercultural de Chiapas" que se desarrolla en la Línea de generación y aplicación del conocimiento: Educación Superior en contextos multiculturales del cuerpo académico: Procesos de Desarrollo Sustentable y Educación Superior Intercultural en la Universidad Intercultural de Chiapas (UNICH).

2 Profesor-investigador en la Universidad Intercultural de Chiapas (UNICH), en la ciudad de San Cristóbal de Las Casas, Chiapas, México. Tiene como Línea de generación y aplicación del conocimiento la Educación Superior en contextos multiculturales. Es miembro del Sistema Nacional de Investigadores del Consejo Nacional de Ciencia y Tecnología (CONACyT). Ocupa el Nivel I. correo electrónico: bastianijose14@hotmail.com

3 Profesora-investigadora en la Universidad Autónoma de Chiapas (UNACH), en Tuxtla Gutiérrez, Chiapas, México. Tiene como Línea de generación y aplicación del conocimiento los procesos de Educación Superior Intercultural. Es miembro del Sistema Nacional de Investigadores del Consejo Nacional de Ciencia y Tecnología (CONACyT). Ocupa el Nivel I. correo electrónico: lpezgm@gmail. com 
desactualizado y es poco pertinente a las necesidades de la población a la que debe atenderse, además responde a una lógica instrumental que afecta profundamente a la formación. Las distintas lenguas de los pueblos originarios no son promovidas como prioridad y tampoco se recupera su cosmovisión. A partir de estos resultados se reflexiona sobre el rumbo de la for- mación del profesorado indígena con el replanteamiento urgente del plan de estudios, acorde al enfoque intercultural que dé sentido y fortalezca la identidad en las comunidades de pueblos originarios.

Palabras clave: educación indígena; formación docente; pueblos originarios; lengua materna; interculturalidad.
The analysis of the teachers' formative process on indigenous education is necessary to revisit the notions of quality, equitable, and contextualized education. For this reason, we report in this paper the results of the research carried out at the Universidad Pedagógica Nacional, Unit 071, in Chiapas, Mexico. This research consisted of an ethnographic study that included the revision of the curricular structure and, in addition, the voice of teachers and students at different training scenarios was taken into account. According to the results, the curriculum is outof-date and irrelevant to the needs of the target population. It was also found that

\section{ABSTRACT}

it responds to an instrumental logic that profoundly affects teachers' formative process.

The different languages of the original peoples are not promoted as a priority and their cosmovision is not recovered either. Based on these results, we reflect on the direction of indigenous teacher training. There is an urgent need to rethink the curriculum from an intercultural perspective that gives value and strength to the identity of the communities of indigenous peoples.

Keywords: indigenous education; teacher training; indigenous peoples; mother tongue; interculturality.

\section{RESUMO}

A análise do processo de formação de professores na educação indígena é necessária para repensar a qualidade da educação com equidade e contextualização. Portanto, os resultados da pesquisa são relatados na Universidade Nacional Pedagógica, Unidade 071, em Chiapas, México. O exercício de pesquisa consistiu em um trabalho etnográfico que incluiu a revisão da estrutura curricular e deu voz a professores e alunos em diferentes momentos e cenários de treinamento. Os resultados indicam que o currículo está desatualizado e não é relevante para as necessidades da população a que deve ser atendido, além de responder a uma lógica instrumental que afeta profundamente o treinamento. As diferentes línguas dos povos nativos não são promovidas como prioridade e sua visão de mundo também não é recuperada. A partir desses resultados, refletimos sobre o curso de formação de professores indígenas com a necessidade de repensar o currículo, de acordo com a abordagem intercultural que dá sentido e fortalece a identidade nas comunidades dos povos nativos

Palavras-chave: educação indígena; formação de professores; povos nativos; língua materna; interculturalidade. 


\section{AMULKÜNU ZUGU}

Müley ta ñi malügeal chum amulen ta kimeltuchefe ñi kimeltugen indígena ñi kimeltuwün mew femgechi ta wüñorakizuamgeay kiñe nor ka kiñe küme kimeltuwün mülelu fey chi mapu mew.

Fey mew, nentugey zugu inaramtun mew Universidad Pedagógica Nacional, Unidad 071, Chiapas, México mapu mew.

Tüfa chi inaramtun zugu mew pepikalgey ta etnográfico küzaw mew chew ta azkintugey estructura curricular ka elugey ñi zugual ta pu kimeltuchefe ka pu chijkatufe fijke antü mew ka mülelu fijke wejin chew ñi kimeltugeken ta che. Wixampüramün mew zugu xipay ta plan de estudios ñi wegenon ka müte kümelay fey chi pu che ñi zuamnien egün, ka fe- mgechi ta wenuntugey kiñe instrumental rakizuam mew fey mew may ta weza amuley kimeltuchen. Fij kake zugun nieulu ta pu pueblos originarios wenuntugekelay ñi kizu egün ni mogen no rume wüñozuamgetukelay. Wixampüramün mew ta müfüke zugu rakizuamgey chem rüpü inageay kimeltugeal ta pu indígena kimeltuchefe ñi matu wüñoazkünugetual ta plan de estudios, koneltu zugun mew ta enfoque intercultural wülal günezugu ka newenuwal ta kizu ñi chegen egün ta pu comunidades de pueblos originarios.

Kimfal hemül: indígena ñi kimeltuwün; kimeltuchen kimeltuchefegeal; pueblos originarios; ñke kewüh, interculturalidad.

\section{INTRODUCCIÓN}

La formación del profesorado para la educación de pueblos originarios es una necesidad apremiante que debe avanzar más allá del discurso de la interculturalidad y atender los aspectos sustantivos atinentes a las condiciones en las que tienen que desarrollarse estas prácticas. En México, y particularmente en el estado de Chiapas, desde hace varias décadas se viene ofreciendo esta formación desde la Universidad Pedagógica Nacional, que tiene como cometido principal fortalecer la formación docente. A la luz de los principales acontecimientos políticos y económicos que han trastocado a los sistemas educativos $y$, en concreto, a la educación de pueblos originarios, se requiere con urgencia la revisión en profundidad del programa de formación de la Licenciatura en Educación Preescolar y Primaria para el Medio Indígena (LEPEPMI), plan 1990, para identificar los retos a los que se enfrenta con los profesores en servicio que laboran con estudiantes de pueblos originarios.

De allí el interés de la investigación que se presenta en este artículo, que tuvo como objetivo conocer las características de la formación docente y profesionalización de los estudiantes de la LEPEPMI'90, así como de su dominio sobre los contenidos del Plan y Programas de Estudio de Educación Básica 2011, específicamente en lo que concerniente a la enseñanza de la lengua indígena. El trabajo, de corte etnográfico, recogió las voces de la muestra de estudiantes y profesores para identificar una serie de situaciones que deben ser atendidas en la búsqueda de dar sentido y rumbo a la formación en el cumplimiento de su perfil de egreso.

En la revisión de trabajos que han abordado la problemática de la formación docente en la educación de pueblos originarios se encontraron elementos comunes en otros ámbitos ya investigados, destacando la importancia que tiene 
la lengua materna y su inclusión en los procesos formativos ${ }^{4}$. Por lo tanto, es necesario repensar desde los diversos escenarios el rumbo de esta formación del profesorado con un intenso trabajo en la resolución de las problemáticas detectadas a lo largo de este estudio y que principalmente pasan por la comprensión de lo que significa la educación de pueblos originarios.

\section{La educación indígena en los programas de la Universidad Pedagógica Nacional}

En el ámbito nacional mexicano, en las décadas de los ochenta y noventa, las demandas históricas y políticas de los pueblos originarios se centraban en la educación indígena bilingüe para atender de forma pertinente y en términos culturales y lingüísticos el desarrollo educativo de estudiantes de las escuelas primarias en las localidades, barrios y parajes indígenas ${ }^{5}$. Las agrupaciones sociopolíticas de campesinos, maestros y maestras bilingües exigían al Estado mexicano propiciar una política educativa que lograra revertir los procesos de castellanización, asimilación e integración como mecanismos de exclusión educativa que aún promovían las escuelas indígenas en pro del eslogan del indigenismo ${ }^{6}$. Estos mecanismos buscaban que en la formación docente se estimulara la integración de las comunidades indígenas a la cultura nacional mexicana. Aun más, la educación bilingüe-bicultural, forjada como acción social, presentaba un proceso pedagógico estrictamente castellanizante, cuya meta era que los estudiantes conocieran y aprendieran la cultura nacional mexicana a través del castellano como única dimensión lingüística para acrecentar el llamado desarrollo económico y cultural de los pueblos originarios, como lo demanda el Estado nacional mexicano; sin embargo, la integración neoliberal de la década de los ochenta, vestida de liberalismo social como proyecto educativo y social nacional, demandaba la construcción de un escenario de competitividad y calidad en la educación indígena, al mismo tiempo que se hacían patentes las exigencias políticas por una educación socioculturalmente pertinente, propuesta por movimientos etno-políticos como el Ejército Zapatista de Liberación Nacional (EZLN) desde 1994, en Chiapas. Cabe mencionar que, como parte de los reacomodos del escenario neoliberal económico, en el año 1992 se realizaron y firmaron los acuerdos para la modernización de la Educación Básica, que implicaban la descentralización y la federalización educativa y, de igual manera, se impulsó

4 Véanse Natalio Hernández Hernández, “Las organizaciones indígenas: ¿autonomía o dependencia?”, en Instituto Nacional Indigenista, 40 años (México: Instituto Nacional Indigenista, 1988), 166-180; Guillermo Bonfil Batalla, "Notas sobre civilización y proyecto nacional", en Instituto Nacional Indigenista, 40 años (México: INI, 1998), 121-140; Margarita Zorrilla Fierro, "Diez años después del Acuerdo Nacional para la Modernización de la Educación Básica en México: Retos, tensiones y perspectivas”, Revista Electrónica de Investigación Educativa, Vol. 4. n.o 2 (2002): 1-19; Graciela Herrera Labra, "Los docentes indígenas. Breve historia", Reencuentro, n.o 33 (2002): 31-39. Rafael Ríos Beltrán. y Martha Cerquera Cuéllar. La modernización de los contenidos y métodos de enseñanza: reflexiones sobre la Escuela Nueva en Colombia. Revista Historia de la Educación Latinoamericana, vol. 16 no. 22, (2014). 157172. https://doi.org/10.19053/01227238.2693

5 UNICACH-UPN, Testimonios Pedagógicos I (México: Universidad de Ciencias y Artes de Chiapas / Universidad Pedagógica Nacional, Unidad 071, 2001); Hernández, "Las organizaciones indígenas".

6 Ibíd.; Bonfil, "Notas sobre civilización”. 
la formación de maestros indígenas en la búsqueda de la eficiencia y la calidad de la educación para abatir el rezago de la educación indígena7.

La coyuntura histórica y política del momento nacional escenificaba la disputa por la constitución de una formación y profesionalización de maestros y maestras indígenas para revertir la deplorable y catastrófica educación de niños y niñas indígenas en el país. Estas condiciones sociopolíticas, que gravitaron en el contexto académico nacional y estatal de forma paralela, incluían en el debate las implicaciones epistemológicas del pluralismo político, la diversidad, el multiculturalismo, los derechos humanos y la misma exigencia del proyecto neoliberal en la educación que se caracterizaba por un fuerte impulso en favor de la calidad, eficiencia y rendición de cuentas en el campo de la educación básica y superior ${ }^{8}$. Esta efervescencia de análisis y cuestionamientos motivó que los actores políticos de la educación indígena reasumieran nuevas formas para construir propuestas curriculares en la formación inicial de profesores para el contexto indígena.

En la Ciudad de México, la Universidad Pedagógica Nacional, Unidad Ajusco, y la Dirección General de Educación Indígena (DGEI) acordaron llevar a cabo un proceso de formación de profesores de educación indígena que, en 1982, bajo la denominación de Licenciatura en Educación Indígena (LEI), tenía como objetivo formar los cuadros académicos para atender pertinentemente la educación de las comunidades indígenas ${ }^{9}$. No obstante, y dados los diversos intereses políticos sobre la academia y por el control del magisterio, se sobre-ideologizaron las intenciones del proyecto de licenciatura, lo que trajo como consecuencia que emergiera otro proyecto formativo que más tarde se convirtió en la Licenciatura en Educación Primaria y Educación Preescolar (en adelante LEPEPMI'90), modelo curricular a seguir en las unidades de la Universidad Pedagógica en el resto

7 Zorrilla, "Diez años después del Acuerdo Nacional”. A diferencia de esta época caracterizada por el auge del neoliberalismo en la que sobrevivía el Instituto Nacional Indigenista cuya visión política era promover, con una visión educativa paternalista, el indigenismo entre las comunidades indígenas, es pertinente señalar que, dos décadas más tarde, el Gobierno Federal promulga el 3 de marzo de 2011, bajo el gobierno del presidente Vicente Fox Quezada, la Ley de la Comisión Nacional para el Desarrollo de los Pueblos Indígenas y se abroga la ley de creación del Instituto Nacional Indigenista; se reforma la fracción VI y se deroga la fracción VII del artículo 32 de la Ley Orgánica de la Administración Pública Federal, y se reforma el primer párrafo del artículo 5.o de la Ley Federal de las Entidades Paraestatales. Esta nueva reglamentación, en pleno auge de las reformas estructurales de naturaleza política y económica impulsadas por el gobierno mexicano bajo el contexto internacional de globalización, redefinió el rumbo de las concepciones de desarrollo social y educativo de las comunidades indígenas de México. Véase http://www.cdi.gob.mx/index.php?option=com_content\&view=article\&id=5\&Itemid=8 (23 de marzo de 2014).

8 Carlos Alberto Torres, “Después de la tormenta neoliberal: La política educativa latinoamericana entre la crítica y la utopía”, Revista Iberoamericana de Educación, n.o 48 (2008): 207-229; Armando Alcántara Santuario, "Políticas educativas y neoliberalismo en México: 1982-2006", Revista Iberoamericana de Educación, n.o 48 (2008): 147-165.

9 Herrera Labra, "Los docentes indígenas". En 1978 la DGEI sostenía convenios de colaboración interinstitucional con el Centro de Estudios sobre Antropología Social (CIESAS), que tenían como objetivo común formar a los cuadros profesionales para promover la educación bilingüe bicultural. En esa coyuntura histórica de finales de la década de los setenta se habían formulado los dos programas curriculares de licenciaturas: Etnolingüística y Ciencias Sociales. Aunque ambos proyectos curriculares fueron construidos, ex profeso, para contribuir a la profesionalización del magisterio indígena, estos tenían fuertes tendencias ideológicas en el ámbito de las relaciones interétnicas y educativas que acabaron por disputarse el control social y político de los maestros y maestras indígenas del país. En Chiapas, un grupo importante se desempeñaba como profesores de primaria indígena, eran líderes políticos y aún ostentan cargos en las dependencias de gobierno, y otros más se han jubilado. En la sede de la Universidad Pedagógica Nacional, Unidad Ajusco México, CDMX, se imparte la licenciatura en Educación Indígena de forma escolarizada, a la que asisten profesores de los 22 estados de la república mexicana a profesionalizarse, como lo sostiene la política formativa de maestros en servicio que posee la Universidad. En la página https://www.upn.mx/index.php/estudiar-en-la-upn/licenciaturas/18-estudiar-en-la-upn/92-educacion-indigena, se localiza información referida a esta licenciatura vigente para la formación y profesionalización docente indígena. 
del país. Este proyecto curricular de y para la formación de maestros y maestras indígenas en el ámbito nacional, experimentó los alcances de las reformas educativas estructurales que promueven la descentralización, el federalismo, la competitividad, la rendición de cuentas, la calidad y evaluación educativa ${ }^{10}$. Con esta licenciatura, la UPN Ajusco en la Ciudad de México (CDMX) se propuso atender la formación docente, en sus 74 sedes y subsedes. Así, a más de dos décadas del origen y desarrollo de la LEPEPMI'90, el escenario de la formación y actualización profesional siguió su curso, experimentando avances y retrocesos. El proceso de construcción del conocimiento formativo se sustentaba en que los estudiantes, que laboran como profesores, pudieran en cuatro años elaborar una propuesta pedagógica como medio para obtener la titulación del programa de licenciatura.

En Chiapas, en la Universidad Pedagógica Nacional, Unidad 071, se había forjado una experiencia formativa para atender el desarrollo profesional de profesores indígenas en las subsedes de la UPN en Palenque, San Cristóbal de Las Casas, Jitotol (antes Bochil), Ocosingo y Las Margaritas, donde se ubican las etnias tsotsil, tseltal, tojolabal, ch'ol y zoque, con las condiciones culturales y lingüísticas necesarias para la profesionalización de maestros bilingües con el objetivo de atender a alumnos indígenas, mediante el Plan UPN $1990^{11}$. A 28 años de haberse constituido en Chiapas como opción profesional, de la LEPEPMI'90 han egresado 6813 profesores bilingües que se han titulado bajo dos modalidades: (a) a través de la propuesta pedagógica y (b) mediante examen realizado por el Centro Nacional de Evaluación para la Educación Superior (CENEVAL). La estadística de fin de curso del ciclo escolar 2012-2013 de la Dirección de Educación Indígena de Chiapas reporta que en las regiones indígenas existen 228093 alumnos inscritos en educación primaria, que presentan un promedio de aprovechamiento general de $7,6 \%$ a nivel estatal. Podemos pensar que esta cifra

10 María de Ibarrola Nicolín y Enrique Bernal Franco, "Descentralización: ¿quién ocupa los espacios educativos? Transformaciones de la oferta escolar de una ciudad mexicana”, Revista Mexicana de Investigación Educativa, Vol. 8, n.o 18 (2003): 379-420.

11 Las nueve regiones económicas que determinó la política de planeación del Gobierno de Chiapas en la década de los ochenta fueron: Centro, Los Altos, Fronteriza, Frailesca, Norte, Selva, Sierra, Soconusco e Istmo-Costa. En la región con población predominante tseltal-tsotsil se localiza la subsede de San Cristóbal; en la región Fronteriza está la subsede de Las Margaritas, con población Tojolabal; en la región Selva está la sede de Ocosingo y Palenque, donde asisten profesores tseltales y choles; en la región Norte está la subsede de Jitotol, donde asisten profesores zoques, choles y tsotsiles, y en la región Centro está la sede principal de Tuxtla Gutiérrez, Chiapas, a donde asisten profesores de filiación zoque, tsotsil, tseltal, tojolabal, entre otros. Como puede verse, las subsedes fueron creadas exprofeso para atender de manera académica la demanda de formación y profesionalización de profesores de educación preescolar y primaria indígena. Esta estrategia ha contribuido a disminuir el ausentismo laboral de profesores y profesoras que laboran en localidades de alta marginación social y en centros educativos ubicados en localidades incomunicadas. En algunas subsedes los inmuebles son arrendados para ser ocupados para las asesorías pedagógicas; la subsede de San Cristóbal no cuenta con edificio propio y la UPN deroga la renta del inmueble; en la subsede de Palenque Chiapas también la Universidad paga la renta; en la subsede de Jitotol se toman las asesorías en las instalaciones del CECYTECH; en la subsede de Margaritas se prestan instalaciones en la escuelas; en la subsede de Ocosingo, con el apoyo económico de alumnos y el respaldo de las autoridades municipales existe un inmueble escolar en dondee concurren los estudiantes-profesores a profesionalizarse; y en la sede Tuxtla se localizan las instalaciones de la Universidad Pedagógica Nacional, Unidad 071. Aunque en el año 2011 hubo otro proceso de regionalización política y económica en donde surgieron quince regiones como divisiones políticas que determinan los niveles de organización territorial y administrativo, la sedes de la UPN y las subsedes continúan ubicadas en regiones estratégicas para contribuir en los procesos de profesionalización docente indígena. Véase la página web [http://www.inafed.gob.mx/work/enciclopedia/EMM07chiapas/regionalizacion.html], fecha de consulta 13 de agosto de 2014 . 
tiene relación con la calidad del proceso de la formación docente recibida en la LEPEPMI'90 por los profesores bilingües ${ }^{12}$.

El trabajo de formación docente de la UPN 071 cuenta con una planta de asesores con diversos perfiles profesiográficos caracterizados, en su mayoría, por una situación laboral de dedicación parcial. Solo cuatro asesores son de tiempo completo de base y están laborando en la sede de Tuxtla Gutiérrez, 18 profesores son de medio tiempo de base, 71 profesores son comisionados por la Subsecretaría de Educación Federalizada, estos últimos adscritos a diferentes modalidades del servicio educativo de educación básica. Además de haber 112 profesores contratados por horas, tanto temporales como de base, para desarrollar actividades de asesoría académica ${ }^{13}$ (UPN, 2014).

Total de profesores de UPN Tuxtla

\begin{tabular}{cc}
\hline Tipo de contratación & Número de profesores \\
\hline Tiempo completo & 4 \\
Medio tiempo & 18 \\
Tiempo parcial & 112 \\
Comisionados & 71 \\
Total & 205 \\
\hline
\end{tabular}

Fuente: Recursos Humanos UPN (2016).

Como se puede observar en la tabla, solo el 1,9\% es de tiempo completo, el $8,5 \%$ es de medio tiempo y el $89,2 \%$ corresponde a profesores de asignatura, situación laboral que puede comprenderse, por una parte, a la situación de indefinición legal-administrativa en el marco de la Modernización de la Educación Básica que en 1992 atravesó la Universidad Pedagógica Nacional en todo el país ${ }^{14}$, y por la otra, a la crisis de empleo que se vive en el país. Esta coyuntura dejó un problema de indefinición jurídica que ha condenado históricamente a la UPN, Unidad 071 a depender administrativamente de la Dirección de Educación Secundaria y Superior de la Subsecretaría de Educación Federalizada sin que, hasta el momento, ostente un techo financiero pertinente para promover la investigación, docencia, difusión y gestión educativa que requiere la formación docente para el medio indígena, en el marco de la política de educación superior en México. Pese al abandono educativo que presenta, la UPN y las subsedes en las regiones de Chiapas han encontrado en la propuesta pedagógica una opción de titulación que permite que los profesores en servicio puedan concluir un ciclo de vida profesional para atender a la educación de estudiantes que viven en las diferentes regiones interculturales de Chiapas.

12 SEF-DEI, "Estadísticas de fin de curso del ciclo escolar 2012-2013", Chiapas: Subsecretaría de Educación Federalizada, Dirección de Educación Indígena, 2013.

13 UPN, “Concentrado general de datos estadísticos del personal académico y administrativo", México: Universidad Pedagógica Nacional, Unidad 071, 2014.

14 UNICACH-UPN, Testimonios Pedagógicos. 


\section{Perspectivas teóricas sobre la formación docente}

Algunas investigaciones señalan que fundamentalmente existen dos perspectivas sobre la formación del profesorado: la técnica y la radical. La primera señala que la enseñanza es una ciencia aplicada y concibe al profesor como un técnico; la segunda implica que la enseñanza sea definida como una actividad crítica y al profesor como un profesional autónomo que realiza investigación sobre su práctica docente. Palomares establece que estas líneas pueden ser especificadas en detalle como la perspectiva académica, la perspectiva técnica, la perspectiva práctica y la perspectiva de reconstrucción social ${ }^{15}$.

La perspectiva académica ${ }^{16}$ en el ámbito de la formación docente sostiene que la enseñanza se circunscribe a la tarea de la transmisión de conocimientos y su adquisición se realiza a través de la enseñanza de la cultura generada por la sociedad. El docente es concebido como un especialista con dominio disciplinar para enseñar los contenidos culturales. Es un intelectual que se forma para transmitir de manera disciplinaria los conocimientos y saberes acumulados social e históricamente. En esta perspectiva se localizan dos enfoques: el enciclopédico y el comprensivo. Estos enfoques aluden a una formación docente que debe concluir con un proceso de alta habilitación académica y de comprensión de los procesos de enseñanza y aprendizaje de la cultura. El enfoque enciclopedista sostiene que, entre más dominio de información tiene el docente, más capacitado está para mejorar y transmitir sus experiencias en la formación de los estudiantes. El profesor es un especialista con responsabilidad académica habilitado para resolver los problemas educativos, ciñe su didáctica a las estructuras lógicas e epistemológicas de las disciplinas. En ese sentido, el trabajo didáctico se circunscribe a homogeneizar la formación porque solamente el docente se aboca a enseñar la tira de materias o asignaturas del programa curricular de la licenciatura.

La perspectiva técnica concibe la formación docente como un proceso de enseñanza de conocimientos que se circunscriben a la aplicación de saberes relacionados con la calidad y a la eficacia ${ }^{17}$. Los resultados de la transmisión de los conocimientos trascienden, siempre y cuando se asuman como portadores de calidad en el producto. El profesor es un técnico que asegura el desarrollo de conocimientos de calidad para el desarrollo de la sociedad. La calidad del conocimiento depende de las especificaciones con que se generan las actividades de investigación y sus componentes epistemológicos como una vía para aplicar y resolver problemas. Schön señala que la perspectiva técnica es una concepción que permite evaluar la enseñanza en los procesos de formación profesional, los

\footnotetext{
15 Ascención Palomares Ruiz, Profesorado y educación para la diversidad en el siglo XXI (Murcia: Universidad de Castilla-La Mancha, 2004).

16 José Gimeno Sacristán y Ángel I. Pérez Gómez, Comprender y transformar la enseñanza (Madrid: Ediciones Morata, 2005).

17 Ibíd.
} 
cuales deben ser eficaces y rigurosos, donde el dominio de la ciencia y las técnicas deben contribuir a la solución de problemas ${ }^{18}$.

Gimeno y Pérez sostienen que los procesos de formación aparecen irradiados por una concepción donde la enseñanza se ha visto como proceso de intervención tecnológica; la investigación como un proceso producto, el maestro como un técnico y la formación del docente dentro del modelo de entrenamiento en competencias $^{19}$. La formación docente por competencias se nutre de dos grandes vertientes de anclaje teórico; la influencia científico-cultural y la psicopedagógica. Ambas dimensiones tienen la finalidad de promover aprendizajes de manera oportuna y eficaz. Estas dos líneas han perfilado una compleja perspectiva técnica de donde se han derivado dos modelos de formación del profesorado. El modelo de entrenamiento y el modelo de adopción de decisiones que implícitamente tienen la intención de preparar al profesional para transmitir conocimiento y resolver problemas con eficacia.

La perspectiva práctica se ha constituido como el modelo más acabado en el diseño curricular para la formación docente. Esta postura sostiene que la enseñanza es una actividad compleja, desarrollada en escenarios singulares determinados por el contexto sociocultural, así como por dinámicas indeterminadas, impredecibles y cargadas de conflictos éticos y políticos. Se concibe al profesor como un artista, un artesano o un profesional clínico para afrontar las diversas situaciones en el aula. Dos han sido los enfoques que han nutrido esta perspectiva: el enfoque tradicional, que se apoya en la experiencia práctica, y el enfoque reflexivo sobre la práctica. En el enfoque tradicional, a pesar de cuestionar la experiencia práctica del profesor, también reconoce que en este proceso no existe un proceso de análisis epistemológico, toda vez que la práctica conlleva un sinnúmero de valores y juicios de las clases sociales hegemónicas que tienen el control del poder. Por lo tanto, la formación del profesor queda subordinada a los vicios y experiencias de las necesidades de las clases que ostentan el poder. La formación profesional del maestro solamente reproduce el orden social determinado.

Por su parte, el enfoque de la reflexión sobre la práctica docente constituye un espacio para democratizar la cultura educativa que aparece subordinada a los lineamientos del ejercicio pedagógico burocrático de dominación tradicional. Este enfoque retoma los postulados de la escuela democrática de Dewey, el cual sostiene que la reflexión sobre la práctica conduce a un proceso de formación científica del maestro sobre su realidad educativa ${ }^{20}$. También Schön insiste en el énfasis pedagógico donde la práctica utiliza la teoría para el análisis de la realidad, en miras de la transformación de las escuelas públicas y la educación

18 Donald Schön, La formación de profesionales reflexivos. Hacia un nuevo diseño de la enseñanza y el aprendizaje en las profesiones (Madrid: Paidós, 1987). http://csmvigo.com/pedagoxia/files/2016/07/D.A.-SCH\%C3\%96N-LA-FORMACI\%C3\%93N-DE-PROFESIONALES-REFLEXIVOS.pdf

19 Gimeno y Pérez, Comprender y transformar.

20 John Dewey, Democracia y educación. Una introducción a la filosofía de la educación (Madrid: Ediciones Morata, 1998). 
de los estudiantes ${ }^{21}$. Este mismo autor señala que hay tres conceptos diferentes que sustentan el pensamiento práctico reflexivo: el conocimiento en la acción, la reflexión en o durante la acción y la reflexión sobre la acción y sobre la reflexión en la acción. Estos tres postulados deben caracterizar los procesos de formación de los profesores en la educación básica. El fin de estos procesos es que el maestro se transforme gradualmente en un investigador para que pueda reflexionar de manera racional su proceso formativo y experiencia en las aulas. Este enfoque permite al profesor atender los problemas educativos de los estudiantes. La reflexión sobre la práctica debe ser una condición necesaria para resolver los problemas de la práctica social y educativa, postulados en común tanto de Kemmis $^{22}$, como Carr $^{23}$ y Habermas ${ }^{24}$.

La perspectiva de reconstrucción social se ha convertido en una alternativa para desarrollar programas de formación docente y se nutre de diversos enfoques y experiencias que sitúan la enseñanza como una actividad crítica y una práctica social saturada de situaciones éticas. El profesional es considerado una figura autónoma que reflexiona críticamente sobre su práctica para comprender el contexto social de la enseñanza y resolver los problemas que se presentan en el aula. En esta corriente se sitúan las aportaciones de Apple ${ }^{25}$, Giroux ${ }^{26}, \mathrm{Kemmis}^{27}$ y Zeichner ${ }^{28}$, quienes coinciden en que los procesos formativos en el campo de la educación deben contribuir a la emancipación de las personas y a construir una democracia participativa y liberal. Esta posición curricular plantea tres grandes procesos: (a) la adquisición de un bagaje cultural de clara orientación política y social; (b) el desarrollo de capacidades de reflexión crítica sobre la práctica y, (c) el desarrollo de actitudes que requieren el compromiso político del profesor como intelectual transformador en el aula, en la escuela y en el contexto social ${ }^{29}$. El enfoque de formación docente asociado con la pedagogía crítica y con la investigación desde la práctica, valora la capacidad de los profesores por producir conocimientos y postula la investigación reflexiva o la enseñanza reflexiva como una propuesta múltiple de enseñanza, aprendizaje e investigación, perfilándose como una opción para mejorar las prácticas formativas de maestros ${ }^{30}$.

Nos preguntamos, y tomando como referencia las anteriores perspectivas, cuál es el modelo que orienta la formación del profesorado para el medio in-

21 Donald Schön, La preparación de profesionales para las demandas de la práctica en la formación de profesionales reflexivos (Barcelona: Paidós, 1992).

22 Stephen Kemmis, El currículum: más allá de la teoría de la reproducción (Madrid: Ediciones Morata, 1998).

23 Wilfred Carr, Una teoría para la educación. Hacia una investigación educativa crítica (Madrid: Ediciones Morata, 2002).

24 Jürgen Habermas, Teoría de la acción comunicativa, II. Crítica de la razón funcionalista (Madrid: Taurus, 1992).

25 Michael Apple, Educación y poder (Madrid: Paidós, 1997).

26 Henry A. Giroux, Los profesores como intelectuales. Hacia una pedagogía crítica del aprendizaje (Madrid: Paidós, 1997).

27 Kemmis, El curriculum: más allá de la teoría.

28 Kenneth M. Zeichner, "El maestro como profesional reflexivo". https://practicareflexiva.pro/wp-content/uploads/2012/04/ Org-El-maestro-como-profesional-reflexivo-de-Kenneth-M.-Zeichner..pdf (20 de octubre de 2018).

29 Palomares, Profesorado y educación.

30 Graciela Messina, "Investigación en o investigación acerca de la formación docente: un estado del arte en los noventa", Revista Iberoamericana de Educación, no. 19 (1999). Enrique Gordillo. Historia de la Educación Mixta y su Difusión en la Educación Formal Occidental. Revista Historia de la Educación Latinoamericana vol. 17 no. 25, (2015): 107-24. https://doi.org/10.19053/01227238.3814. 
dígena. Siendo así, a continuación, se presenta un apartado que, en voz de los participantes, nos aproxima a reconocer sus percepciones sobre el desarrollo de la experiencia docente en el ámbito de la formación de profesores de educación primaria y preescolar indígena.

\section{Metodología}

El objetivo general de este ejercicio de investigación fue conocer las características de la formación docente y profesionalización de los estudiantes de la LEPEPMI'90, así como de su dominio sobre los contenidos del Plan y Programas de Estudio de Educación Básica 2011, específicamente en lo que toca a la enseñanza de la lengua indígena. Se utilizó la perspectiva cualitativa, que desde el punto de vista de Cañón, Monrroy y Salcedo ${ }^{31}$ permite atender a las subjetividades a partir de los contextos como forma de indagar la cotidianidad y las dinámicas de interacción. Por ello el método de investigación fué la etnografía, que permitió la descripción, comprensión e interpretación de las acciones de los sujetos en el contexto escolar ${ }^{32}$ formativo como lo es la Universidad Pedagógica Nacional, Unidad 071, de Tuxtla Gutiérrez, Chiapas. Se utilizaron diversas estrategias de recolección de datos; en primer lugar, se analizó la estructura curricular del Plan 90 de la LEPEPMI; en segundo lugar, se asistió a las reuniones de academia y, en tercer lugar, se entrevistó de manera colectiva a una muestra del estudiantado, de acuerdo con criterios de inclusión que serán presentados más adelante.

Se propició la inclusión fisica en las reuniones de los asesores para el trabajo colegiado, las cuales se constituyeron en el espacio pedagógico que permitió captar las percepciones que de forma verbal construían y denotaban en las sesiones de trabajo los profesores. En total se llevaron a cabo cinco reuniones a lo largo del ciclo escolar 2016 (enero-junio). Se prestó especial atención a la academia de profesores de 7. y 8. o semestres. Así también se revisaron las minutas de dichas reuniones para identificar los juicios de los profesores acerca de los contenidos, metodologías de trabajo, adecuaciones curriculares que consideraban necesarias, las sesiones de trabajo de fin de semestre a través del Taller Integrador y la Propuesta Pedagógica, los problemas administrativos, las formas de evaluación de los alumnos y alumnas, la desaparición gradual de la LEPEPMI'90, la emergencia de nuevas propuestas curriculares, el cumplimiento de los contenidos de los diversos programas de estudio, la organización institucional, la participación de los asesores en los procesos de movilización sindical, las condiciones laborales de la planta de asesores ${ }^{33}$.

La sede Tuxtla, compuesta por una población de cien estudiantes y once profesores de asignatura, fue el escenario de investigación. En el caso de las

31 Lilia Cañón Flórez, Myriam Esperanza Monroy González y Javier Ricardo Salcedo. Experiencia pedagógica y voz multicultural: pasos y palabras en historia de vida de maestra afrodescendiente. En http://www.scielo.org.co/pdf/rhel/v18n27/v18n27a08.pdf

32 James McKernan, Investigación-acción y currículum (Madrid: Ediciones Morata, 1996).

33 Paul Croll, La observación sistemática en el aula (Madrid: Editorial La Muralla, 1995); Raúl Rojas Soriano, Guía para realizar investigaciones sociales (México: Plaza y Valdez Editores, 2002). 
observaciones a la planta docente a través del trabajo cooperativo se hizo con la totalidad de los profesores y en el caso de las entrevistas a los estudiantes, se realizó con una muestra del 11 \% que se encontraban cursando el octavo semestre. Los criterios de inclusión fueron los siguientes: (a) ser profesor en servicio, (b) hablante de una lengua materna distinta al español y (c) ser oriundo de un pueblo originario del estado de Chiapas. 36,3\% hablan tseltal, 36,3\% hablan tsoltsil y 27,2 \% hablan cho' 1 .

Los once profesores que laboran en el programa educativo son de asignatura, siete $(63,6 \%)$ tienen un posgrado y el resto solo cuenta con licenciatura. Del total, cinco $(45,4 \%)$ se encuentran en el rango de quince a veinte años de experiencia en docencia en el nivel superior y seis $(54,5 \%)$ tienen de cinco a diez años como docentes. Se indagó sobre su experiencia docente con el programa, su concepción del programa de formación y profesionalización, el modelo y enfoque curricular empleado para la elaboración de la propuesta pedagógica, así como su condición laboral.

En las entrevistas colectivas sostenidas con el estudiantado se abordaron como temas centrales los siguientes:

- Sus expectativas de formación docente a partir de los cursos recibidos durante este proceso.

- Su experiencia con respecto a la construcción de la propuesta pedagógica como medio de titulación, la enseñanza de la lengua indígena y el castellano.

- La comprensión de la educación básica.

- La percepción sobre los asesores.

- El impacto de esta formación en su práctica docente cotidiana.

Así mismo, se hizo el análisis del plan de la Licenciatura para la revisión de contenidos en sus programas de estudio para identificar la pertinencia de la formación en el contexto indígena.

Las entrevistas realizadas a los estudiantes fueron de carácter colectivo, haciendo énfasis además en la trayectoria que han recorrido para insertarse en el mundo occidentalizado a través de la escuela o desde algún otro ámbito como el laboral. Estas entrevistas (cuatro) se llevaron a cabo en las aulas de la UPN sede Tuxtla, con una duración aproximada de dos horas cada una durante los meses de mayo y junio de 2016. Los estudiantes no permitieron que fueran grabadas por ningún medio, registrándose en el diario de campo con la ayuda del entrevistador y dos observadores.

\section{Resultados}

a) El plan 90 de la LEPEPMI, su origen y su estructura curricular. El Plan y Programa de Estudio de la LEPEPMI'90 fue elaborado por un grupo de profesionales con diversas disciplinas académicas en la Ciudad de México, con la participación de profesores de las sedes y subsedes de UPN del país. Ese grupo académico, a través de un proceso de investigación, elaboró el documento curricular para hacerlo operable en las 72 sedes y subsedes de la UPN en el país; 
con la concepción de que en las sedes de UPN de los estados pudieran realizarse las adecuaciones curriculares para atender de manera específica los entornos socioculturales de los maestros tsotsiles, tseltales, ch'oles, tojolabales y zoques en Chiapas, para contribuir en la mejora de calidad de la educación indígena.

El Plan y Programa de Estudio de la LEPEPMI'90 tiene como perfil de egreso las siguientes consideraciones:

\begin{abstract}
Será un profesional de la docencia y deberá para el desarrollo de su práctica docente considerar las características psicológicas de los niños y del contexto sociocultural, su práctica docente como objeto de conocimiento y de transformación pedagógica, problematice su trabajo pedagógico otorgándole especificidad a su profesión, posea una sólida formación metodológica y teórica que apoyado en las aportaciones de las distintas disciplinas pueda construir propuestas pedagógicas, conocimientos psicopedagógicos que fomenten el desarrollo de aprendizajes significativos, dominio de conocimientos socio históricos que le permita analizar su práctica docente con respecto a la política educativa nacional, conocimientos teóricos y metodológicos desde la perspectiva antropológico lingüística para reconocer la lengua y la cultura de la comunidad, incorpora los conocimientos culturales de la comunidad indígena sin menoscabo de lo nacional y universal, cuenta con elementos teóricos metodológicos para mejorar su práctica docente y fortalece desde la escuela la lengua indígena y el español. ${ }^{34}$
\end{abstract}

El programa de la LEPEPMI'90 se encuentra estructurado por campos y líneas disciplinarias articulados a partir de cursos que definen de manera gradual el desarrollo de la formación docente de maestros en servicio del medio indígena. La organización curricular horizontal está conformada por un área de formación denominada básica y otra de formación terminal. En el área básica convergen cuatro líneas que paralelamente incentivan la reflexión sobre la práctica docente. En esta área curricular se pretende generar un proceso de habilitación a maestros para que construyan, a partir de veinte cursos, la Propuesta Pedagógica, la cual es el producto eje de formación y para su logro se organizó a partir de cinco líneas formativas: (a) psicopedagógica, (b) antropológico-lingüística, (c) socio-histórica y (d) metodológica ${ }^{35}$. Cada una de estas tiene el objetivo de desarrollar habilidades y estrategias cognoscitivas para que el estudiante avance en su formación de manera escalonada, asegurando el dominio de aprendizajes de contenidos en los cursos para apuntalar de manera integral la elaboración de la Propuesta Pedagógica como trabajo final.

Una estrategia importante también lo es la socialización de los contenidos curriculares para proveer una experiencia diversa sobre los aprendizajes de tipo teórico y metodológico para constituir el acervo necesario que propicie la reflexión sobre la práctica docente. Finalmente, otra estrategia es el trabajo en el área terminal, donde las líneas de formación convergen de manera integral,

34 UPN, Plan y Programa de Estudio de la Licenciatura en Educación Preescolar y Primaria para el Medio Indígena, Plan 1990 (LEPEPMI'90) (México: Universidad Pedagógica Nacional, 1990).

35 Ibíd. 
desembocando en cuatro campos formativos: (a) social, (b) lengua, (c) matemática y $(\mathrm{d})$ naturaleza ${ }^{36}$. Estos "campos" tienen relación con las asignaturas que se imparten en los programas de los niveles educativos: educación preescolar y primaria indígena, Consejo Nacional para el Fomento de la Educación (CONAFE) y Proyecto Educador Comunitario Indígena (PECI). En estos campos formativos, los profesores-estudiantes en servicio elaborarán durante el sexto semestre una propuesta de estrategia didáctica por cada campo. La intención es que, para el séptimo semestre, la propuesta se presente de manera ordenada decantándose como una Propuesta Pedagógica con fines de titulación. Cabe mencionar que el programa de estudio, elaborado en la década de los noventa para todo el país, tiene relación con el Plan y Programas de Estudio de Educación Básica primaria 1993, que concebía para su logro los aprendizajes a partir de objetivos ${ }^{37}$. Durante la formación docente se enfatiza que los aprendizajes son por objetivos y, por ello, los cursos tienen objetivos que definen de manera gradual los alcances de aprendizaje de cada alumno, en cada semestre de la formación básica y terminal.

En Chiapas, "el currículum de la LEPEPMI'90 se reorganizó parcialmente a partir de las necesidades de los maestros y alumnos, intentando tomar en cuenta sus características culturales y lingüísticas" (3. e reunión de academia, 2016). No obstante, esta realidad étnica en lo curricular ha sido rebasada por las complejas transformaciones socioculturales de las regiones indígenas, donde convergen problemas añejos como la pobreza, migración tanto interna como externa, analfabetismo, pérdida de recursos bióticos, expulsión por motivos religiosos, tráfico de drogas, entre otros. Es necesario realizar una revisión curricular donde la Propuesta Pedagógica pueda retomar o considerar estos problemas que se relacionan con las acciones docentes y necesidades áulicas de los profesores indígenas de las regiones. Hasta el momento de la redacción de este artículo, el programa educativo no ha sufrido ningún cambio curricular. Siendo este el panorama político sobre la formación del profesorado para el medio indígena, nos preguntamos desde qué perspectiva teórica se están formando para construir una Propuesta Pedagógica.

b) Los asesores y el trabajo colegiado. La mirada del profesorado es importante para la operación del programa de forma tal que, desde hace más de diez años, se han organizado en academias en las que se vierten sus concepciones y sus preocupaciones, dando lugar a la identificación de la forma en que perciben también el plan de estudios. Esta organización por academias debe dar lugar a lo que se denomina trabajo colegiado que, como se verá más adelante, se encuentra con un número importante de retos que deben ser superados con la mayor brevedad posible.

36 Ibíd.

37 Véase el documento SEP, Plan y Programas de Estudio de Educación Básica Primaria, 1993 (México: SEP CDMX). A lo largo de las prescripciones de cada asignatura aparecen los objetivos de las unidades de contenidos para su socialización en el aula como objetivos de aprendizajes que se deben alcanzar en el ciclo escolar que dispone el desarrollo de los planes y programas de estudio de educación primaria. 
El trabajo colegiado, desde el punto de vista de Espinosa ${ }^{38}$, son las relaciones educativas que ejecutan las personas cara a cara, de mutuo apoyo y de colaboración con un fin único que permite potenciar los analisis y atender las situaciones de trabajo escolar en la educación superior. En ese mismo sentido, se plantea que el trabajo colegiado implica intercambios comunicativos y una importante interacción, ambos necesarios para los aprendizajes y solución de problemas ${ }^{39}$ en las reuniones de los asesores académicos de la LEPEPMI'90.

El plan de estudios de la LEPEPMI'90 fue elaborado por un grupo de destacados profesores de la UPN de la Unidad Ajusco, con el apoyo de los profesores de las sedes en el país. Se reconoce que fue un trabajo importante llevado a cabo en todo el país para atender las exigencias coyunturales políticas y académicas durante la década de los noventa. Sin embargo, los profesores destacan que "los alcances de la LEPEPMI'90, en materia de contenidos, no están en sintonía con los enfoques por competencias, las formas de organización escolar y las demandas de habilidades digitales que exige el Plan y programas de Estudio 2011 de Educación Básica producto de la Reforma Integral de la Educación Básica llevadas a cabo entre los años 2004 y 2011, en México" (2.. reunión de academia).

Otra de las preocupaciones de los profesores que fungen como asesores se relaciona con la construcción de la Propuesta Pedagógica como estrategia de formación y titulación, razón por la cual es el tema recurrente en las reuniones a lo largo de los semestres. Los asesores académicos insisten en que su papel es muy importante para la formación del estudiantado y consideran que podrían desarrollar mejor su práctica docente si contaran con un posgrado.

Por otra parte, no existe consenso acerca de las características de este trabajo de Propuesta Pedagógica, ni el modelo a seguir para su desarrollo. Con respecto a la estructura a manera de procedimientos que debe seguirse para la construcción de la propuesta, un $30 \%$ de los profesores coincide en señalar que primero el estudiante debe hacer un diagnóstico, para después plantear el problema estrictamente educativo de aprendizaje hasta llegar al domino conceptual y construir la propuesta metodológica didáctica que incluya los criterios de evaluación. El resto de los asesores, que corresponde al $70 \%$, sostiene que debe iniciarse con el planteamiento del problema para desarrollar posteriormente la propuesta. Desde su punto de vista, es necesario en principio plantear el problema y como consecuencia construir la propuesta desde los referentes teóricos y didácticos para llevarla al aula primaria y preescolar. Además, los asesores confunden el dominio teórico con respecto a la práctica docente y las implicaciones de ésta aplicada a los problemas centrales en la Propuesta Pedagógica. Aparece la teoría desarticulada de la realidad educativa. En general se logra identificar que entre los asesores prevalece una confusión del paradigma constructivista y otras corrientes teóricas que tienen relación en todos los campos formativos y

38 María Eugenia Espinosa Carbajal, "El trabajo colegiado en las escuelas normales y la evaluación de sus planes de estudio", CPU-e, Revista de Investigación Educativa, n.o 7, (2008): 1-14.

39 Frida Díaz Barriga y Gerardo Hernández Rojas, Estrategias docentes para un aprendizaje significativo. Una interpretación constructivista (México: Editorial McGraw-Hill, 2010). 
problemas sin que haya una relación específica con los problemas educativos reales que experimentan los alumnos en las escuelas de organización multigrado o completo en las regiones indígenas.

Los asesores presentan un dominio teórico de corrientes y paradigmas en el campo del constructivismo, pero no logran articular sus esfuerzos conceptuales en explicar las implicaciones abstractas a los problemas que presentan los alumnos en los diferentes campos específicos de formación de la LEPEPMI'90. En el ejercicio de la formación docente que impulsan los asesores se observan trabajos con poca vinculación de las implicaciones de contenidos de los cursos que imparten para su aplicación y uso en las propuestas pedagógicas. Así mismo, en las asesorías no se generan actividades metacognitivas de articulación entre la teoría y la práctica donde se sitúan los niveles de intervención pedagógica de las estrategias didácticas que serán aplicados en la solución de problemas de aprendizaje entre los alumnos. Además, prevalece un error de concepción curricular en el papel de los asesores con respecto a la elaboración de la Propuesta Pedagógica porque no se considera como una investigación científica, sino como un dispositivo técnico que contribuye a resolver problemas prácticos en el aula.

Aunque en el mismo currículo de la LEPEPMI'90 toda la información de contenidos que aparece en los programas de estudio enfatiza el dominio científico para el desarrollo de la Propuesta Pedagógica, en la práctica se observa en los asesores una carencia de estrategias didácticas para la formación en investigación porque las asesorías se convierten en experiencias de trabajo grupal de manera mecánica que desembocan en el aburrimiento y abandono de la elaboración de la propuesta por parte de los alumnos. Es de llamar la atención que una buena parte de la planta de asesores presenta poco o nulo dominio de la lengua indígena, mucho menos la escriben, aunque la LEPEPMI'90 señala en el perfil de egreso que los maestros alumnos se graduarán con una concepción de valoración de la identidad lingüística.

Para el caso de la LEPEPMI, y debido a que el total de los profesores es contratado como de asignatura (horas/clase), estos consideran que solo deben cumplir con las horas contratadas para las asesorías y por lo tanto el trabajo de supervisión de las propuestas no es pobible. Los asesores aceptan que prevalece un futuro de incertidumbre laboral pues hace varias décadas el gobierno los tiene en el olvido educativo y social porque no se hacen contrataciones de nuevas plazas de tiempo completo. Al respecto, un profesor con diez años de antigüedad señala lo siguiente:

Tengo casi veinte años trabajando como profesor de asignatura y no veo para cuándo me contraten por lo menos como medio tiempo; me parece que me voy a jubilar y no lograré alcanzar ese estatus. La verdad eso me desmotiva temporalmente pero luego pienso que así me tocó vivir, me pongo las pilas y sigo trabajando. ${ }^{40}$

40 José Bastiani Gómez y María Minerva López García, Diario de campo, Universidad Intercultural de Chiapas, San Cristóbal de Las Casas, Chiapas, México, febrero de 2016. 
También los procesos de descentralización de la educación superior de la década de los noventa han implicado que la UPN siga sin un estatuto orgánico legal como entidad de educación superior que cuente con techo financiero y promueva los cambios institucionales requeridos por la realidad educativa en el estado y el país. Por otra parte, conciben a la licenciatura como un programa que ha llegado a su fin porque hay pocos alumnos o profesores en servicio que han seguido este camino de la profesionalización. Sugieren nuevas opciones curriculares aunque la dependencia de UPN Ajusco, México, imposibilita el proceso curricular de nuevos programas de forma local. Señalan los asesores académicos que han egresado muy buenos profesores indígenas, con una sólida formación docente que les ha permitido concluir con una Propuesta Pedagógica, pero al mismo tiempo existen estudiantes que aún no saben planear sus clases con dificultades metodológicas para elaborar este trabajo y titularse en tiempo y forma de la UPN porque no cubren con el perfil, es decir, no son profesores en servicio sino estudiantes de tiempo completo.

\section{La experiencia formativa del estudiantado}

El estudiantado, en su mayoría, admite que se sigue sosteniendo un currículo en apariencia pertinente a las necesidades culturales y lingüísticas de los estudiantes, maestros y comunidades indígenas, dada la denominación con la que se bautizó en su origen; sin embargo, señalan que en la práctica el desenlace curricular en el campo de la formación docente de forma individual y colectiva se ha traducido en un proceso de homogenización educativa de las identidades étnicas. Un estudiante comenta lo siguiente:

\footnotetext{
Me siento decepcionado, pensaba que iba a aprender a enseñar en mi propia lengua a mis estudiantes. Yo hablo tseltal y cuando llego al salón veo que mis profesores no lo hablan, quieren que hablemos español y no hay ninguna diferencia de cómo debo tratar a mis alumnos. Sí que me preocupa porque escogí esta licenciatura para que yo diera algo bueno a mi comunidad y eso no va a pasar. ${ }^{41}$
}

Una de sus mayores preocupaciones está en el hecho de que debe impartirse desde las lenguas tzeltal, tsotsil, zoque y español, pero la competencia en español se privilegia empleándose para elaborar el documento recepcional con serias dificultades para la redacción. Como consecuencia, tampoco se concreta la lengua indígena como elemento transversal en la Propuesta Pedagógica.

Otro aspecto nodal es el relacionado con la concreción de la Propuesta Pedagógica, debido a que aún en semestres avanzados no logran comprender cómo deben realizar el trabajo. Con respecto a esta idea, un estudiante señaló que:

$41 \quad$ Ibíd., enero de 2016. 
No entiendo bien lo de la propuesta, la hemos venido trabajando desde casi que empezamos la carrera pero unos profesores nos piden una cosa, luego vienen otros y nos piden una diferente a lo que ya hicimos y entonces no se les entiende. Casi todos en mi salón estamos confundidos, no sabemos a quién de los profesores hacerle caso. Y pregúnteme, ¿qué estamos aprendiendo?: nada. Luego piensan en pura escuela urbana castellanizante y esa no es nuestra realidad. Quizá sea para los compañeros que viven en la ciudad y que no son profesores indígenas pero no es el caso de nosotros. ${ }^{42}$

De allí que el estudiantado, en su mayoría, considera que la formación adquirida en el programa de la LEPEPMI'90 no les permite avanzar lo suficiente para elaborar este trabajo de propuesta debido a que impera en sus trayectorias escolares el modelo de una escuela urbana castellanizante que admite la existencia de comunidades y escuelas indígenas con una visión de mundo mesoamericano, lo cual limita seriamente su actuación en el contexto de formación para el mundo indígena. En esta misma dimensión, el estudiantado ubica al castellano como una limitante que no les permite avanzar en su formación para la enseñanza de escuelas bilingües. En la práctica cotidiana de cada quince días de asistencia a la UPN, el alumnado señala que se promueve poco la reflexión sobre su práctica docente del profesor indígena y se quedan en el plano de la teoría del mundo occidentalizado, con pocos asesores formados para comprender lo que implica este trabajo de diseño y aplicación de la propuesta y reconocen que los existentes tienen una habilitación académica insuficiente y poco alfabetizados en su lengua materna para recuperar saberes y valores de las comunidades indígenas que contribuyan a la construcción de la ruta metodológica para la elaboración de la Propuesta Pedagógica con un buen nivel de aplicación y resultados con los alumnos de primaria indígena.

Por otra parte, un factor que también afecta su formación son sus condiciones laborales, con sobrecarga de trabajo como profesor en servicio en actividades necesarias de planeación, reunión con padres, traslado a las comunidades y, en algunos casos, sin luz eléctrica.

\section{Discusión (un escenario prospectivo para la formación del profesorado)}

Dos tendencias relacionadas con los procesos de formación docente han marcado las investigaciones en el ámbito del diseño curricular: el técnico racional y el de sentido. En la LEPEPMI'90 se debe permitir que las diversas concepciones puedan ser revisadas para estructurar un nuevo planteamiento curricular que, a través de enfoques, modelos y perspectivas, conlleven a la elaboración de un programa curricular de y para los pueblos originarios de Chiapas. Los fundamentos teóricos, filosóficos y políticos del Plan y Programas de Estudio de la LEPEPMI'90 fueron conformados por un modelo curricular tradicional que responde a las disciplinas centradas en la enseñanza y aprendizaje de los

42 Ibíd., marzo de 2016. 
alumnos. Aunque al desarrollo curricular le han agregado enfoques como el de la investigación-acción, entre otros, para mejorar la calidad del trabajo que realiza el profesor indígena, también es cierto que las experiencias de los estudiantes-alumnos presentan resultados poco alentadores en su formación al no traducirse en avances significativos en cuanto a la calidad de los aprendizajes de los alumnos indígenas.

La experiencia de formación y profesionalización de maestros y maestras indígenas a partir de la LEPEPMI'90 presenta una construcción curricular que se ubica en un período marcado por los influjos de la conformación del Estado nacional mexicano y por una amplia relación económica capitalista que ha subsumido a la práctica de formación docente. Es así que la citada formación recibida por los profesores indígenas no ha podido redefinir la educación de los pueblos indígenas.

La LEPEPMI'90 se constituyó en una experiencia novedosa para la formación docente indígena, pero sus alcances de profesionalización se han caracterizado por seguir promoviendo una práctica indigenista, integracionista y de asimilación cultural, lo que no ha permitido una formación docente de y para las comunidades indígenas. Esta formación ya no responde a los señalamientos curriculares del Plan y Programas de Estudio 2011 de Educación Básica, el cual es producto de la Reforma Integral de la Educación Básica (RIEB). Además, la LEPEPMI'90, dado el contexto universitario en el que se desarrolla y la indefinición jurídica que presenta la UPN, propicia que las prácticas formativas realizadas por los asesores se queden en el terreno de lo intencional y de buenos sueños porque priva la incapacidad en los niveles de articulación, los diferentes paradigmas pedagógicos y los niveles de intervención educativa (Propuesta Pedagógica) en las escuelas indígenas.

Por otra parte, y dadas las circunstancias de no ofrecer opciones de profesionalización en el reconocimiento de la lengua materna, se puede afirmar que predomina una visión de la formación del profesor subordinada a las clases con un profesor que reproduce el orden social determinado.

Es así como el conjunto de intenciones programáticas curriculares no logra desarrollarse entre los maestros-alumnos y el malestar se convierte en una condición más o menos permanente, tanto de los estudiantes como de los asesores, porque coexiste una relación burocrática que se inserta en un contexto local, regional, nacional e internacional capitalista ${ }^{43}$. La crisis moral que se presenta en los procesos de formación a partir de la LEPEPMI'90 entre estudiantes y asesores académicos es una crisis del aparato burocrático que fue alimentada por el nacionalismo y el capitalismo mexicanos, bajo la lógica del indigenismo y que ahora requiere de un proceso de renovación curricular por competencias como lo demanda la RIEB. La reingeniería curricular que demanda el Estado mexicano moderno, a través de la política educativa indígena, se inserta en una reestructuración burocrática liberal del sistema educativo que exige el cumpli-

43 Emilio Tenti Fanfani, “Consideraciones sociológicas sobre profesionalización docente”, Educação y Sociedade, Vol. 28, n.o 99 (2007): 335-253; Thomas S. Popkewitz, Sociología política de las reformas educativas (Madrid: Ediciones Morata, 2000). 
miento del Plan y Programa de Estudio 2011de Educación Básica, estructurado bajo el paradigma racional técnico, lo que requiere de un programa de formación docente de corte management (cliente-alumno) para atender la educación de los pueblos originarios ${ }^{44}$. Es así como, en la década de los noventa, se constituyó en la época del debate académico y político sobre temas de pluralismo, diversidad, interculturalidad, género, sustentabilidad, entre otros, que a nivel de formación docente fueron alimentados por una tendencia "intercultural" en la LEPEPMI'90 de forma secundaria y se ha traducido en una experiencia insuficiente para atender el desarrollo de las lenguas y culturas originarias para la construcción de ciudadanías interculturales ${ }^{45}$. La escasez de recursos financieros para la investigación debida a la irregularidad jurídica-laboral como parte del entramado burocrático organizacional de la UPN no ha permitido que la investigación se haya consolidado como plataforma indispensable para resolver los problemas educativos de la formación docente y la educación en las regiones indígenas de Chiapas.

\section{CONCLUSIÓN}

El trabajo de renovación institucional que debe realizar la UPN en el campo de la formación y profesionalización de maestros y maestras indígenas requiere ser enfrentado a partir de dimensiones institucionales para ejercer una gobernanza política de la educación superior intercultural. En los campos de la gestión y la gobernanza de políticas educativas interculturales, las autoridades y asesores académicos, además de las representaciones político sindicales, deben encaminarse bajo una agenda de trabajo, la demanda de reconocimiento jurídico de la UPN frente a las instancias de los gobiernos estatal y nacional para que en un plazo de tiempo considerable se sienten las bases de desarrollo de una Universidad. Esto demanda un proceso de reorganización académico-administrativa centrada en: la construcción de Líneas de Generación y Aplicación del Conocimiento (LGAC), contratación de profesores de tiempo completo en la sede de Tuxtla Gutiérrez y subsedes, Cuerpos Académicos, programa de desarrollo profesional con alto nivel de habilitación académica y la asignación de un presupuesto financiero acorde con las normas que prescribe el Consejo Nacional de Ciencia y Tecnología (CONACYT) y la Secretaría de Educación Pública (SEP). La urgencia de un nuevo planteamiento curricular requiere de la participación de profesores indígenas con amplia trayectoria académica donde se privilegie el uso de la lengua indígena, así como su cosmovisión con una revisión profunda de las diversas epistemologías, filosofías, conocimientos, saberes y literaturas

44 Emilio Tenti Fanfani, “Consideraciones sociológicas sobre profesionalización docente”, Educaçao y Sociedade, Vol. 28, n.o 29 (2007): 335-253.

45 Luis Enrique López Hurtado, “Democracia y cambios en la educación indígena en América Latina: a manera de epílogo", en La EIB en América Latina bajo examen, eds. Luis Enrique López y Carlos Rojas (La Paz: Banco Mundial-GTZ, Plural Editores, 2006), 367373; Ruth Moya, "La interculturalidad para todos en América Latina", en Interculturalidad, educación y ciudadanía. Perspectivas latinoamericanas, ed. Luis Enrique López (La Paz: FUNPROEIB Andes - Ediciones Plural, 2009), 21-57. 
que estructuran la realidad de los pueblos tsotsiles, choles, tseltales, tojolabales y zoques, entre otros, para ser incorporados en el planteamiento curricular. Además de revisar los paradigmas, modelos y enfoques de formación docente que provienen de las dos tradiciones: el racional técnico y el de sentido.

\section{FUENTES}

Entrevista a Miguel Velázquez, José Crisógono, estudiante del octavo semestre de la Licenciatura en Educacion Primaria y Preescolar para el Medio Indígena (LEPEMI). Universidad Pedagógica Nacional, Unidad 071, Tuxtla Gutiérrez, Chiapas, México, 22 de febrero, 25 de abril, 18 de mayo, 6 de junio de 2016.

Entrevista a Pérez Rodríguez, Domingo Oliverio, estudiante del octavo semestre de la LEPEMI. Universidad Pedagógica Nacional, Unidad 071, Tuxtla Gutiérrez, Chiapas, México, 22 de febrero, 25 de abril, 18 de mayo, 6 de junio de 2016.

Entrevista a Estrada, María de los Ángeles, estudiante del octavo semestre de la LEPEMI. Universidad Pedagógica Nacional, Unidad 071, Tuxtla Gutiérrez, Chiapas, México. 22 de febrero, 25 de abril, 18 de mayo, 6 de junio de 2016.

Entrevista a Flores Hernández, Amalia, estudiante del octavo semestre de la LEPEMI. Universidad Pedagógica Nacional, Unidad 071, Tuxtla Gutiérrez, Chiapas, México. 22 de febrero, 25 de abril, 18 de mayo, 6 de junio de 2016.

Entrevista a Gómez de la Cruz, Fermín, estudiante del octavo semestre de la LEPEMI. Universidad Pedagógica Nacional, Unidad 071, Tuxtla Gutiérrez, Chiapas, México. 22 de febrero, 25 de abril, 18 de mayo, 6 de junio de 2016.

Entrevista a Gómez Gómez, José Clever, estudiante del octavo semestre de la LEPEMI. Universidad Pedagógica Nacional, Unidad 071, Tuxtla Gutiérrez, Chiapas, México. 22 de febrero, 25 de abril, 18 de mayo, 6 de junio de 2016.

Entrevista a Gómez Pérez, Adelina, estudiante del octavo semestre de la LEPEMI. Universidad Pedagógica Nacional, Unidad 071, Tuxtla Gutiérrez, Chiapas, México. 22 de febrero, 25 de abril, 18 de mayo, 6 de junio de 2016.

Entrevista a López Vázquez, Beatriz, estudiante del octavo semestre de la LEPEMI. Universidad Pedagógica Nacional, Unidad 071, Tuxtla Gutiérrez, Chiapas, México. 22 de febrero, 25 de abril, 18 de mayo, 6 de junio de 2016.

Entrevista a Morales Vázquez, Silvia del Rosario, estudiante del octavo semestre de la LEPEMI. Universidad Pedagógica Nacional, Unidad 071, Tuxtla Gutiérrez, Chiapas, México. 22 de febrero, 25 de abril, 18 de mayo, 6 de junio de 2016.

Entrevista a Pérez Hernández, María, estudiante del octavo semestre de la LEPEMI. Universidad Pedagógica Nacional, Unidad 071, Tuxtla Gutiérrez, Chiapas, México. 22 de febrero, 25 de abril, 18 de mayo, 6 de junio de 2016.

Entrevista a Santiz Méndez, Dalia Esther, estudiante del octavo semestre de la LEPEMI. Universidad Pedagógica Nacional, Unidad 071, Tuxtla Gutiérrez, Chiapas, México. 22 de febrero, 25 de abril, 18 de mayo, 6 de junio de 2016.

\section{REFERENCIAS}

Alcántara Santuario, Armando. "Políticas educativas y neoliberalismo en México: 1982-2006". Revista Iberoamericana de Educación, n. ${ }^{\circ} 48$ (2008): 147-165.

Apple, Michael. Educación y poder. Madrid: Paidós, 1997.

Bastiani Gómez, José y María Minerva López García. Diario de campo. Universidad Intercultural de Chiapas, San Cristóbal de Las Casas, Chiapas, México, enero de 2016. 
Bastiani Gómez, José y María Minerva López García. Diario de campo. Universidad Intercultural de Chiapas, San Cristóbal de Las Casas, Chiapas, México, febrero de 2016.

Bastiani Gómez, José y María Minerva López García. Diario de campo. Universidad Intercultural de Chiapas, San Cristóbal de Las Casas, Chiapas, México, marzo de 2016b.

Bonfil Batalla, Guillermo. "Notas sobre civilización y proyecto nacional”. En Instituto Nacional Indigenista 40 años. México: INI, 1998.

Carr, Wilfred. Una teoría para la educación. Hacia una investigación educativa crítica. Madrid: Ediciones Morata, 2002.

Congreso de los Estados Unidos Mexicanos. Ley de la Comisión Nacional para el Desarrollo de los Pueblos Indígenas. Poder Ejecutivo Federal, Gobierno de los Estados Unidos Mexicanos. http://www.cdi.gob.mx/index.php?option=com_content\&view=article\&id=5\&Itemid=8

Croll, Paul. La observación sistemática en el aula. Madrid: Editorial La Muralla, 1995.

De Ibarrola Nicolín, María y Enrique Bernal Franco. “Descentralización: ¿quién ocupa los espacios educativos? Transformaciones de la oferta escolar de una ciudad mexicana". Revista Mexicana de Investigación Educativa 8, n. ${ }^{\circ} 18$ (2003): 379-420.

Dewey, John. Democracia y educación. Una introducción a la filosofía de la educación. Madrid: Ediciones Morata, 1998.

Díaz Barriga, Frida y Gerardo Hernández Rojas. Estrategias docentes para un aprendizaje significativo. Una interpretación constructivista. México: Editorial McGraw-Hill, 2010.

Espinosa Carbajal, María Eugenia. “El trabajo colegiado en las escuelas normales y la evaluación de sus planes de estudio". CPU-e, Revista de Investigación Educativa, n. 7 (2008): 1-14.

Gimeno Sacristán, José y Ángel I. Pérez Gómez. Comprender y transformar la enseñanza. Madrid: Ediciones Morata, 2005.

Giroux, Henry A. Los profesores como intelectuales. Hacia una pedagogía crítica del aprendizaje. Madrid: Paidós, 1997.

Gordillo, Enrique. Historia de la Educación Mixta y su Difusión en la Educación Formal Occidental. Revista Historia de la Educación Latinoamericana vol. 17 no. 25, (2015): 107-24. https://doi. org/10.19053/01227238.3814.

Habermas, Jürgen. Teoría de la acción comunicativa, II. Crítica de la razón funcionalista. Madrid: Taurus, 1992.

Hernández Hernández, Natalio. “Las organizaciones indígenas: ¿autonomía o dependencia?”. En Instituto Nacional Indigenista 40 años. México D. F.: INI, 1988.

Herrera Labra, Graciela. “Los docentes indígenas. Breve historia". Reencuentro, n. ${ }^{\circ} 33$, (2002): 31-39.

Kemmis, Stephen. El currículum: más allá de la teoría de la reproducción. Madrid: Ediciones Morata, 1998.

López Hurtado, Luis Enrique. “Democracia y cambios en la educación indígena en América latina: a manera de epílogo". En La EIB en América Latina bajo examen, editado por Luis Enrique López y Carlos Rojas. La Paz: Banco Mundial-GTZ, Plural Editores, 2006, 367-373.

McKernan, James. Investigación-acción y curriculum. Madrid: Ediciones Morata, 1996.

Messina, Graciela. "Investigación en o investigación acerca de la formación docente: un estado del arte en los noventa". Revista Iberoamericana de Educación, n. ${ }^{\circ} 19$ (1999).

Moya, Ruth. "La interculturalidad para todos en América Latina". En Interculturalidad, educación y ciudadanía. Perspectivas Latinoamericanas, editado por Luis Enrique López. La Paz: FUNPROEIB Andes / Ediciones Plural, 2009, 21-57.

Palomares Ruiz, Ascención. Profesorado y educación para la diversidad en el siglo XXI. Murcia: Universidad de Castilla-La Mancha, 2004.

Popkewitz, Thomas S. Sociología política de las reformas educativas. Madrid: Ediciones Morata, 2000.

Ríos Beltrán, R. y Cerquera Cuéllar, M. La modernización de los contenidos y métodos de enseñanza: reflexiones sobre la Escuela Nueva en Colombia. Revista Historia de la Educación Latinoamericana, vol. 16 no. 22, (2014). 157-172. https://doi.org/10.19053/01227238.2693

Rojas Soriano, Raúl. Guía para realizar investigaciones sociales. México: Plaza y Valdez Editores, 2002.

Schein, Edgar H. Organizational Culture and Leadership. EE.UU.: Jossey Bass Books, 2010. http://books.google.es/books?hl=es\&lr=\&id=kZH6AwTwZV8C\&oi=fnd\&pg=PR9\&d$\mathrm{q}=$ Schein+(1992)+\&ots=9nb_nCAzLj\&sig=zsUqP1a5ji3qZW-HddjK_SuvjZg\#v=onep- 


\section{Universidad Pedagógica Nacional, Unidad 071, de Chiapas}

age\&q=Schein $\% 20(1992) \& \mathrm{f}=$ false

Schön, Donald. La formación de profesionales reflexivos. Hacia un nuevo diseño de la enseñanza y el aprendizaje en las profesiones. Madrid: Paidós, 1987.

Schön, Donald. "La preparación de profesionales para las demandas de la práctica". En La formación de profesionales reflexivos. Barcelona: Paidós, 1992. Recuperado de: http://csmvigo. com/pedagoxia/files/2016/07/D.A.-SCH\%C3\%96N-LA-FORMACI\%C3\%93N-DE-PROFESIONALES-REFLEXIVOS.pdf

SEF-DEI. "Estadísticas de fin de curso del ciclo escolar 2012-2013". Chiapas: Subsecretaría de Educación Federalizada, Dirección de Educación Indígena, 2013.

SEP. “Planes y Programas Educativos de Educación Primaria de 1993”. México: Secretaría de Educación Pública, 1993.

SEP. “Plan de Estudios 2011. Educación Básica”. México: Secretaría de Educación Pública, 2011.

Stenhouse, Lawrence. Investigación y desarrollo del currículum. Madrid: Ediciones Morata, 2003. http://books.google.es/books?id=TzGPp8411_AC\&printsec=frontcover\&dq=Stenhouse,+1985\&hl=es\&sa $=$ X\&ei=vOwgVLuYE8r-8AHu5IHYBg\&ved $=0$ CE8Q6AEwBg\#v=onepage\&q\&f=false.

Tenti Fanfani, Emilio. “Consideraciones sociológicas sobre profesionalización docente". Educação y Sociedade 28, n. ${ }^{\circ} 99$ (2007): 335-253.

Torres, Carlos Alberto. “Después de la tormenta neoliberal: La política educativa latinoamericana entre la crítica y la utopía". Revista Iberoamericana de Educación, n. ${ }^{\circ} 8$ (2008): 207-229.

UNICACH-UPN. Testimonios Pedagógicos I. México: Universidad de Ciencias y Artes de Chiapas / Universidad Pedagógica Nacional, Unidad 071, 2001.

UPN. Concentrado general de datos estadísticos del personal académico y administrativo. México: Universidad Pedagógica Nacional, Unidad 071, 2014.

UPN. Plan y Programa de Estudio de la Licenciatura en Educación Preescolar y Primaria para el Medio Indígena, Plan 1990 (LEPEPMI'90). México: Universidad Pedagógica Nacional, 1990.

Zeichner, Kenneth M. "El maestro como profesional reflexivo". https://practicareflexiva.pro/ wp-content/uploads/2012/04/Org-El-maestro-como-profesional-reflexivo-de-Kenneth-M.-Zeichner..pdf (20/10/2018).

Zorrilla Fierro, Margarita. “Diez años después del Acuerdo Nacional para la Modernización de la Educación Básica en México: Retos, tensiones y perspectivas". Revista Electrónica de Investigación Educativa 4, n. ${ }^{\circ} 2$ (2002): 1-19.

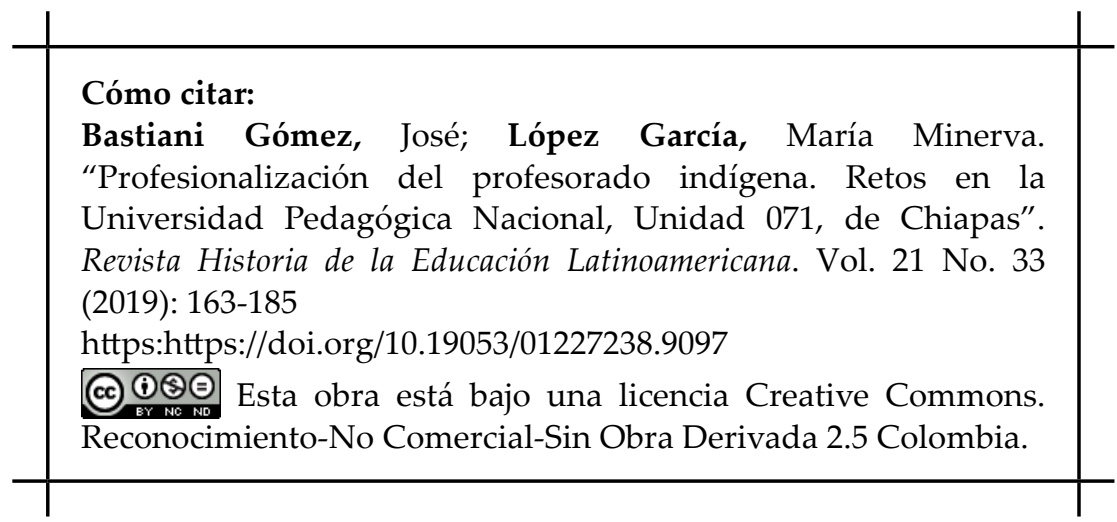

